\title{
Recent Development in Fabrication of Co Nanostructures and Their Carbon Nanocomposites for Electromagnetic Wave Absorption
}

\author{
Nannan $\mathrm{Wu},{ }^{1, *}$ Wenjing Du, ${ }^{2}$ Qian $\mathrm{Hu},{ }^{3, *}$ Sravanthi Vupputuri ${ }^{3}$ and Qinglong Jiang ${ }^{4, *}$
}

\begin{abstract}
In recent years, Electromagnetic wave (EMW) absorption materials have received great interests owing to their ability of addressing the increased EM radiation (EMR) and interference (EMI) problems. With the advantages of high magnification saturation and large permeability in $\mathrm{GHz}$ range, cobalt materials have been considered as promising EM absorbers. This article introduces the development of pure Co materials with different micro-structures and Co/C composites as EM wave absorption materials. The effects of various microstructures, filling ratios, and graphitization degree on the EM wave absorption properties were thoroughly discussed. Through comparisons of different types of Co based absorbers, the combination of Co and porous carbon can be an effective method and main direction to reach the requirements of ideal EMW absorption materials with strong absorption, low thickness, broad absorption bandwidth and low density.
\end{abstract}

Keywords: Electromagnetic Wave Absorption; Cobalt; Carbon Materials; Magnetic Loss; Dielectric Loss.

Received: 3 September 2020; Accepted: 23 October 2020.

Article type: Review article.

\section{Introduction}

In the past decades, electronic devices such as cell phones, iPads, and laptops have been widely employed in civil applications. ${ }^{[1]}$ Zhang reported that under the electromagnetic field, the drug delivery could be achieved. ${ }^{[2]}$ As electromagnetic field brings great convenience to people's life, it also leads to serious and electromagnetic wave interference (EMI) problems that not only influences the operation of electrical devices but also severely affects people's health. ${ }^{[3-5]}$ The key solution to address this critical issue is to develop high-efficiency EM wave absorption materials, which are

${ }^{1}$ Department of Materials Science and Engineering, Shandong University of Science and Technology, Qingdao, Shandong 250061, China

${ }^{2}$ College of Materials Engineering, Henan Key Laboratory of Electronic Ceramics Materials and Application, Henan University of Engineering, Zhengzhou, 451191, China

${ }^{3}$ Department of Chemical \& Biomolecular Engineering, University of Tennessee, 1512 Middle Drive, Knoxville, Tennessee 37996, USA

${ }^{4}$ Department of Chemistry and Physics, University of Arkansas, Pine Bluff, Arkansas 71601, USA

* Email: wunanan1991@163.com (N. Wu); qhu10@utk.edu (Q. Hu); jiangq@uapb.edu (Q. Jiang) capable of converting incident EM waves into thermal energythrough their magnetic or dielectric loss ability. ${ }^{[6-13]}$ Ideal EM wave absorbers (EMAs) should meet the demands of lightweight, strong absorption, thin thickness and broad absorbing bandwidth. Among the various kinds of EM wave absorption materials, the ferromagnetic metals, especially cobalt $(\mathrm{Co})$ materials have attracted great interests because of their unique features including large saturation magnetization, high Snoek's limit, compatible dielectric loss and distinguishable permeability in $\mathrm{GHz}$ frequency range. ${ }^{[14-18]}$ Owing to their benefits, the number of publications concerning about the investigations of Co material as EM wave absorbents were increasing over the last decades. However, previous researches suggested that pure Co materials always suffer from many drawbacks such as high density, impedance mismatch and rapid decrease of permeability in high frequency range due to the eddy current effect (the alternated magnetic field induced by eddy current can cancel the external magnetic field and lead to decreased permeability), which will weaken their EM wave absorption ability. ${ }^{[19-21]}$

Up to now, there are two effective solutions to overcome these shortcomings and improve the absorbing performances. The first one is to design Co absorbers with microstructural 

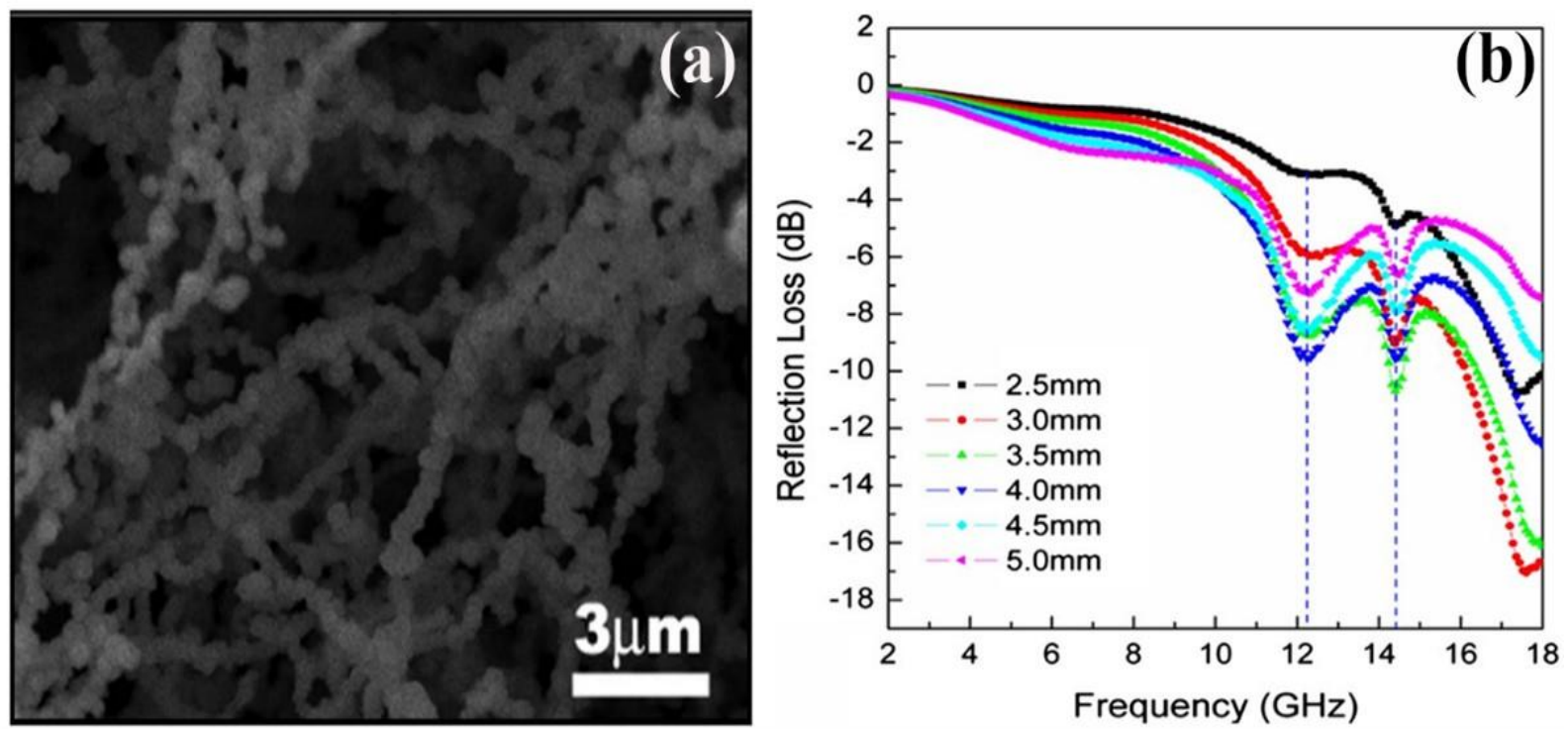

Fig. 1 (a) SEM image of the hollow Co nanochains and (b) calculated RL curves of hollow Co nanochains versus frequency and thickness, reproduced with the Permission from [39]. Copyright@American Institute of Physics.

features such as a size less than skin depth, low density and large surface area. ${ }^{[19]}$ For example, flower-like Co superstructures composed of leaf-like flakes exhibited a minimum reflection loss $\left(\mathrm{RL}_{\min }\right)$ of $-40.25 \mathrm{~dB}$ at $6.08 \mathrm{GHz}$ with a thickness of $2.5 \mathrm{~mm} \cdot{ }^{[22]}$ A porous three-dimensional Co@CoO flowers were synthesized by Lv et al and obtained outstanding EM wave absorption properties with an optimal $\mathrm{RL}$ of $-50 \mathrm{~dB}$ and the effective bandwidth (RL $<-10 \mathrm{~dB}$, corresponding to $90 \%$ EM wave absorption) was up to $11 \mathrm{GHz}$ at $2.0 \mathrm{~mm} \cdot{ }^{[20]} \mathrm{He}$ and co-workers synthesized the hollow porous Co spheres successfully, which presented wide absorption frequency range of $4.0-12.0 \mathrm{GHz}(\mathrm{RL}<-20 \mathrm{~dB}$, corresponding to $99 \%$ EM wave absorption) in the thickness ranged from 1.4 to $4.0 \mathrm{~mm}$ and the excellent EM wave absorption was attributed to better impedance matching of the hollow structures. ${ }^{[16]}$ These results indicated that microstructures have a significant effect on the EM wave absorption properties of the absorbers. The other solution to improve EM wave absorbing performances of Co materials is to hybridize cobalt particles with dielectric materials (including $\mathrm{SiO}_{2}, \mathrm{TiO}_{2}$, carbon materials, polymers, etc.) to increase the dielectric loss ability and optimize EM parameters. ${ }^{[23,24]}$ Zhang et al synthesized carbon-encapsulated cobalt nanoparticles successfully and obtained a minimum RL of $-52 \mathrm{~dB}$ at $7.54 \mathrm{GHz}$ for the $\mathrm{Co}(\mathrm{C})$ composites. ${ }^{[25]}$ The enhanced EM wave absorption was ascribed to the improved anti-oxidization ability and impedance matching. The microporous core-shell Co@CoO nanoparticles fabricated by Liu et al exhibited quite high RL value of $-90.2 \mathrm{~dB}$ and a wide broad absorption bandwidth of $7.2 \mathrm{GHz} .{ }^{[26]}$ The hierarchical $\mathrm{Co} / \mathrm{C}$ crabapples synthesized by Wu et al showed broad-band absorption with the bandwidth of $5.9 \mathrm{GHz}$ at an ultrathin thickness of $1.4 \mathrm{~mm} \cdot{ }^{[27]}$ Recently, metal-organic-frameworks (MOFs) derived porous carbon/Co nanocomposites derived through pyrolysis of ZIF-67 have attracted great attentions and excellent EM wave absorption was reported. ${ }^{[28-30]}$

Considering of these new developments, it is quite necessary to provide a critical assessment and future applications of Co-based materials as EM wave absorbers. To the best of our knowledge, reviews concerned about
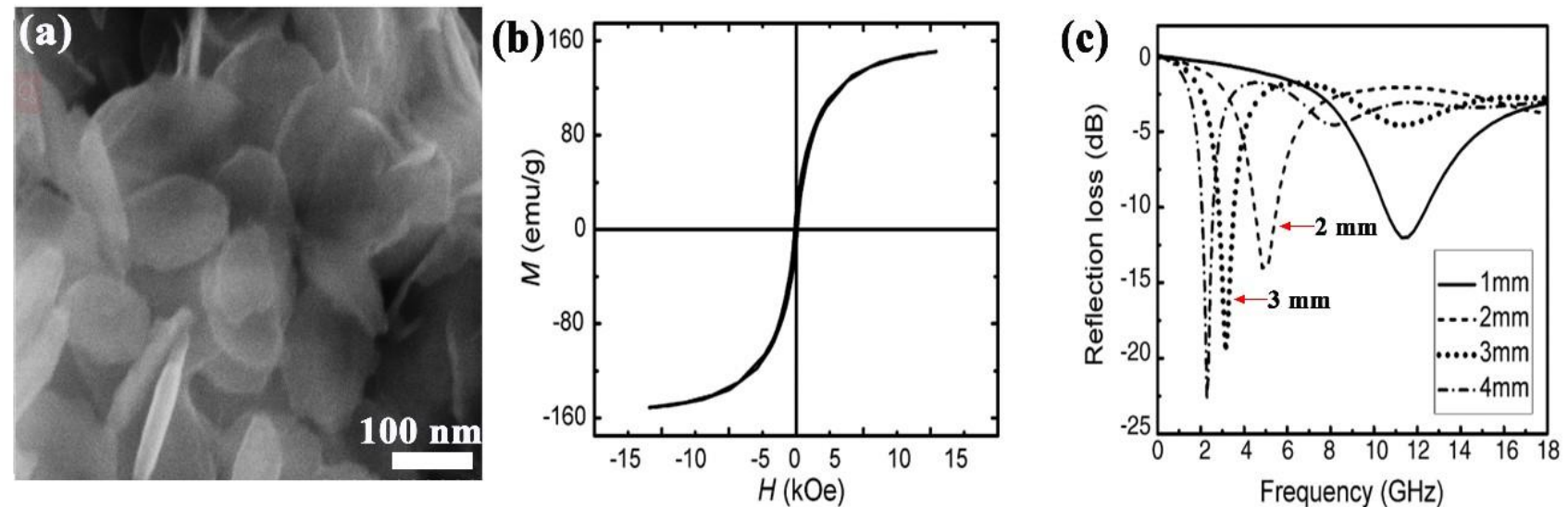

Fig. 2 (a) SEM image, (b) magnetic hysteresis loop and (c) RL values with frequency and thicknesses for the Co nanoplatelets, reproduced with the Permission from [43]. Copyright@ 

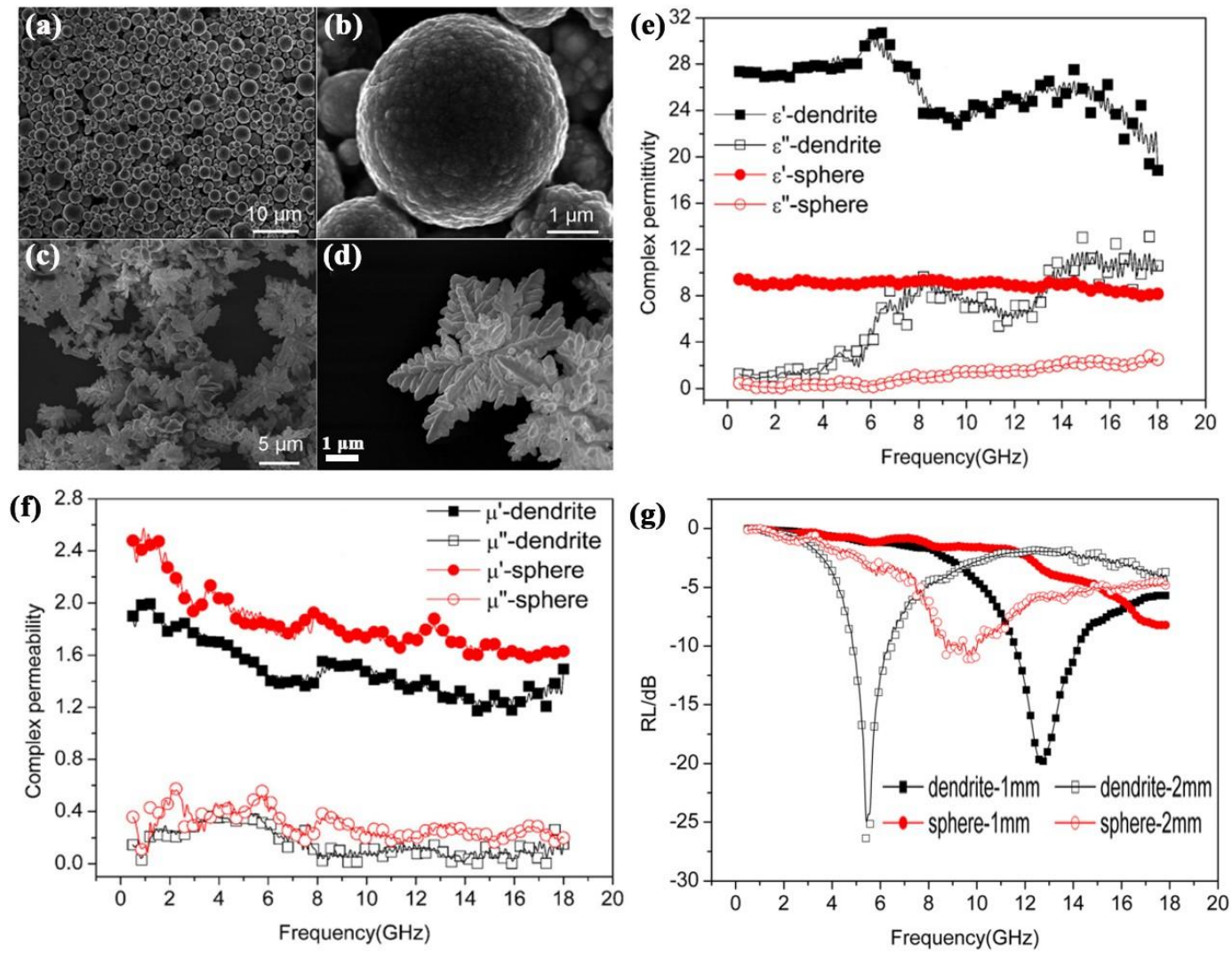

Fig. 3 (a) SEM images of the spherical Co at low magnification and (b) high magnification; SEM images of the dendritic Co at low magnification (c) and (d) high magnification. Frequency dependence of (e) complex permittivity, (f) complex permeability and (g) RL values of the dendritic and spherical samples in 2-18 GHz, reproduced with the Permission from [49].

development of Co-based absorbers haven't been outlined. This review focuses on the progress of Co materials and their composites as highly efficient EM wave absorption materials. Then the effects of microstructures and components on the EM wave absorption of Co-based materials along with the related attenuation mechanisms are discussed in detail. Finally, the challenges and prospects of Co-based materials for EM wave absorption applications are concluded.

\section{Co-based Materials as EMAs}

\section{1 pure Co materials as EMAs}

Due to their excellent magnetic loss ability resulting from the large saturation magnetization $\left(168 \mathrm{emu} \mathrm{g}^{-1}\right.$ for the bulk cobalt), pure Co materials are considered as potential EMAs. ${ }^{[16]}$ However, two main shortcomings of pure Co restrict their wide application as EMAs. Firstly, the large conductivity of metallic Co can result in a much larger $\varepsilon_{r}$ value according to the free electron theory $\varepsilon^{\prime \prime} \approx \sigma /\left(2 \pi \varepsilon_{0} f\right),{ }^{[31]}$ which causes the mismatch between $\varepsilon_{r}$ and $\mu_{r}$. Secondly, the permeability of pure magnetic metals will drop rapidly in high frequency range owing to the eddy current effect, which causes a weak EM wave absorption. ${ }^{[32-34]}$ Designing special microstructures, such as porous, hollow and flake-like structures, has been proved to be an effective way to regulate the EM parameters and achieve impedance matching. ${ }^{\left[{ }^{[3-38]}\right.}$ In this section, the pure Co materials as EMAs will be discussed and the influences of microstructures on the EM wave absorption properties of pure Co EMAs are clarified.

\subsubsection{One-dimensional (1D) and two-dimensional (2D) Co materials as EMAs}

The EM wave absorption performances of 1D and 2D Co materials have been widely reported in early studies. In Shi's report, the 1D hollow Co nanochains with length of tens of micrometers were synthesized and the paraffin wax composites containing $20 \mathrm{wt} \%$ Co nanochains showed a $\mathrm{RL}_{\text {min }}$ of $-17.1 \mathrm{~dB}$ at $17.5 \mathrm{GHz}$ with the thickness of $3.0 \mathrm{~mm}$ (Fig. 1a and b). ${ }^{[39]}$ The one-dimensional Co nanowires synthesized by Chen et al exhibited $\mathrm{RL}_{\min }$ of $-23.5 \mathrm{~dB}$ at $6.5 \mathrm{GHz}$ with the thickness of $5.0 \mathrm{~mm}$ (Fig. 1c and d). ${ }^{[40]}$ As discussed above, these previously reported 1D Co EMAs displayed efficient EM wave absorption to an extent, however, the absorption peaks below $-10 \mathrm{~dB}$ is only observed at a certain frequency with differnet thicknesses. Thus, effective absorption of wide frequency range can't be obtained. According to Walser's theoretical analysis and experimental investigations, the large shape anisotropy of flake-shaped particles enable it to exceed the Snoek's limit and own a larger permeability in $\mathrm{GHz}$ range. ${ }^{[41,42]}$ In the following studies, Ma et al reported the EM properties of Co nanoflakes and owing to the large shape anisotropy, surface anisotropy and exchange energy of Co nanoflakes, multiple resonance peaks were observed in the 

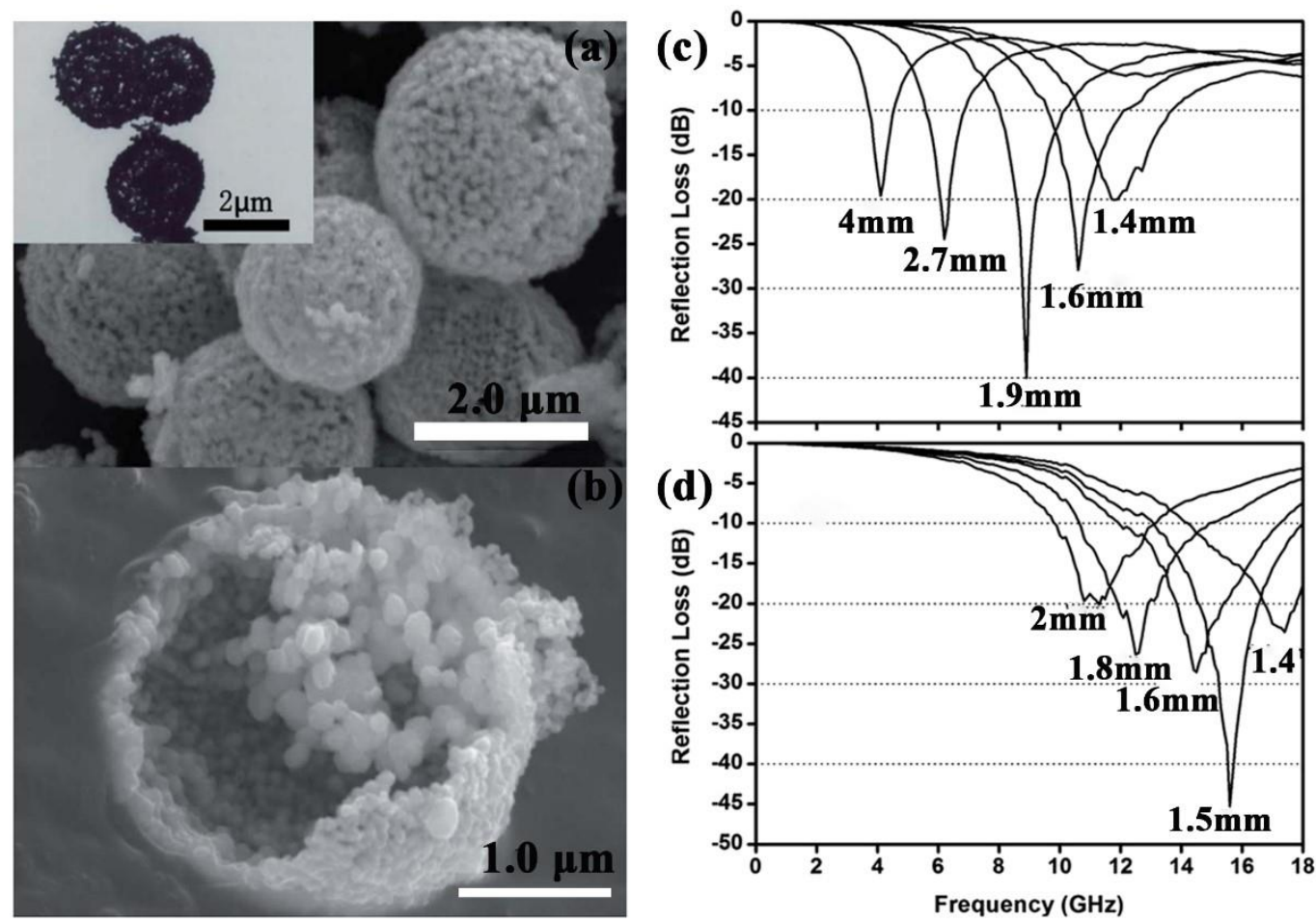

Fig. 4 SEM image of (a) Co microspheres and (b) cracked Co spheres; Frequency dependence of reflection loss for the epoxy resin composites containing (e) $60 \mathrm{wt} \%$ and (f) $30 \mathrm{wt} \%$ hollow Co spheres, reproduced with the Permission from [16].

permeability-frequency curves, indicating the 2D flakeshaped Co could be considered as a broadband candidate for EM wave absorption. ${ }^{[15]} \mathrm{Li}$ et al investigated the magnetic properties and EM wave absorption performances of Co nanoplatelets in 0.1-18.0 GHz. ${ }^{[43]}$ As shown in Fig. 2a and b, the coercivity of Co nanoplatelet is $109 \mathrm{Oe}$, which is much larger than the bulk Co (ca. 10 Oe). Effective absorption is achieved in broad frequency range of $2-11.5 \mathrm{GHz}$ in the thickness ranges of 1.0-4.0 mm (Fig. 2c). These results suggest that the flake-shaped microstructure is beneficial to improve the magnetic loss and broaden the absorption bandwidth.

\subsubsection{Three-dimensional (3D) Co materials as EMAs}

In the past decades, more and more attentions are attracted to develop 3D hierarchical structural Co materials (e.g. flowerlike, dendritic-like, sword-like etc) as EMAs. ${ }^{[19,20,44]}$ On one hand, the large surface area of hierarchical structures is beneficial to generate multiple dielectric polarizations (such as interface polarizations and dipole polarizations). ${ }^{[45,46]}$ On the other hand, the 3D structure could induce the production of multiple reflections and scatterings, through which the incident EM waves can be attenuated gradually. ${ }^{[47,48]}$ Liu et al investigated the effect of hierarchical architecture on the microwave absorption properties of $\mathrm{Co} /$ paraffin wax composites. ${ }^{[49]}$ As shown in Fig. 3a-d, the spherical and dendritic Co with diameters of 1.0-5.0 $\mu \mathrm{m}$ were synthesized respectively and their EM parameters were measured. Comparing with Co spheres, the dendritic Co displayed more fluctuations and larger level of permittivity, as can be seen in Fig. $3 \mathrm{e}$ and $3 \mathrm{f}$, inferring the enhanced polarization ability of
Co than sphere-like Co. The massive branches of Co dendrites were favorable to form large number of interfaces between the fillers and paraffin wax, causing the difference of polarization processes. On account of the enhanced dielectric loss ability, the Co dendrites presented obviously stronger EM wave absorption than Co spheres as observed in Fig. 3g. Flowerlike Co structures composed of numerous leaf-like flakes were prepared by Tong et al. ${ }^{[22]}$ When the weight ratio of Co powders and paraffin wax is $2: 1$, the $\mathrm{RL}_{\min }$ achieved -40.25 $\mathrm{dB}$ and the absorption bandwidth with RL less than $-20 \mathrm{~dB}$ ( $99 \%$ absorption) reached $13.28 \mathrm{GHz}$ in the thickness ranges of 1.0-4.0 $\mathrm{mm}$. Wang investigated the electromagnetic properties of cobalt assemblies with different morphologies, including Co spheres and Co flowers with sharp petals. ${ }^{[50]}$ Comparing with sphere-like Co, the Co flowers with sharp petals exhibited enhanced EM wave absorption performance and the enhancement is associated with the morphological advantages of flower-like structures. The numerous petals of Co flowers can be served as wires, which induced the incident EM wave energy into dissipative current along the wire that resulted in attenuation. He and co-workers have synthesized the porous Co hollow microspheres with diameters of 2-4 $\mu \mathrm{m}$ through combining a solvothermal route with chemical reduction process (Fig. 4a and b). ${ }^{[16]}$ For the epoxy resin composites containing $60 \mathrm{wt} \%$ Co hollow microspheres, the strongest peak of $-40 \mathrm{~dB}$ appeared at $9.0 \mathrm{GHz}$ with the matching thickness of $1.9 \mathrm{~mm}$ and wide absorption frequency range from 4.0-12.0 $\mathrm{GHz}$ was obtained with the thickness ranges of 1.4-4.0 mm (Fig. 4c). For the samples with $30 \mathrm{wt} \%$ Co hollow microspheres, the optimal RL value reached -45 

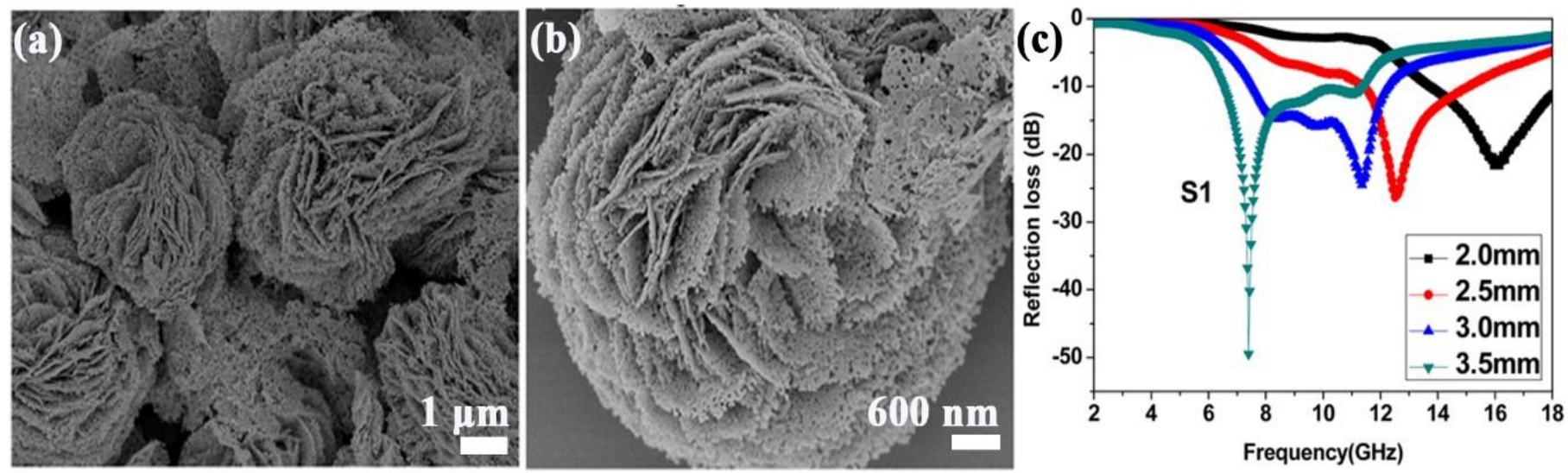

Fig. 5 (a, b) SEM images of porous 3D flower-like $\mathrm{Co} / \mathrm{CoO}$ with different magnifications; (c) RL values of the $\mathrm{Co} / \mathrm{CoO}$ samples at different thickness; (d) The counter map of RL for the $\mathrm{Co} / \mathrm{CoO}$ samples, reproduced with the Permission from [20].
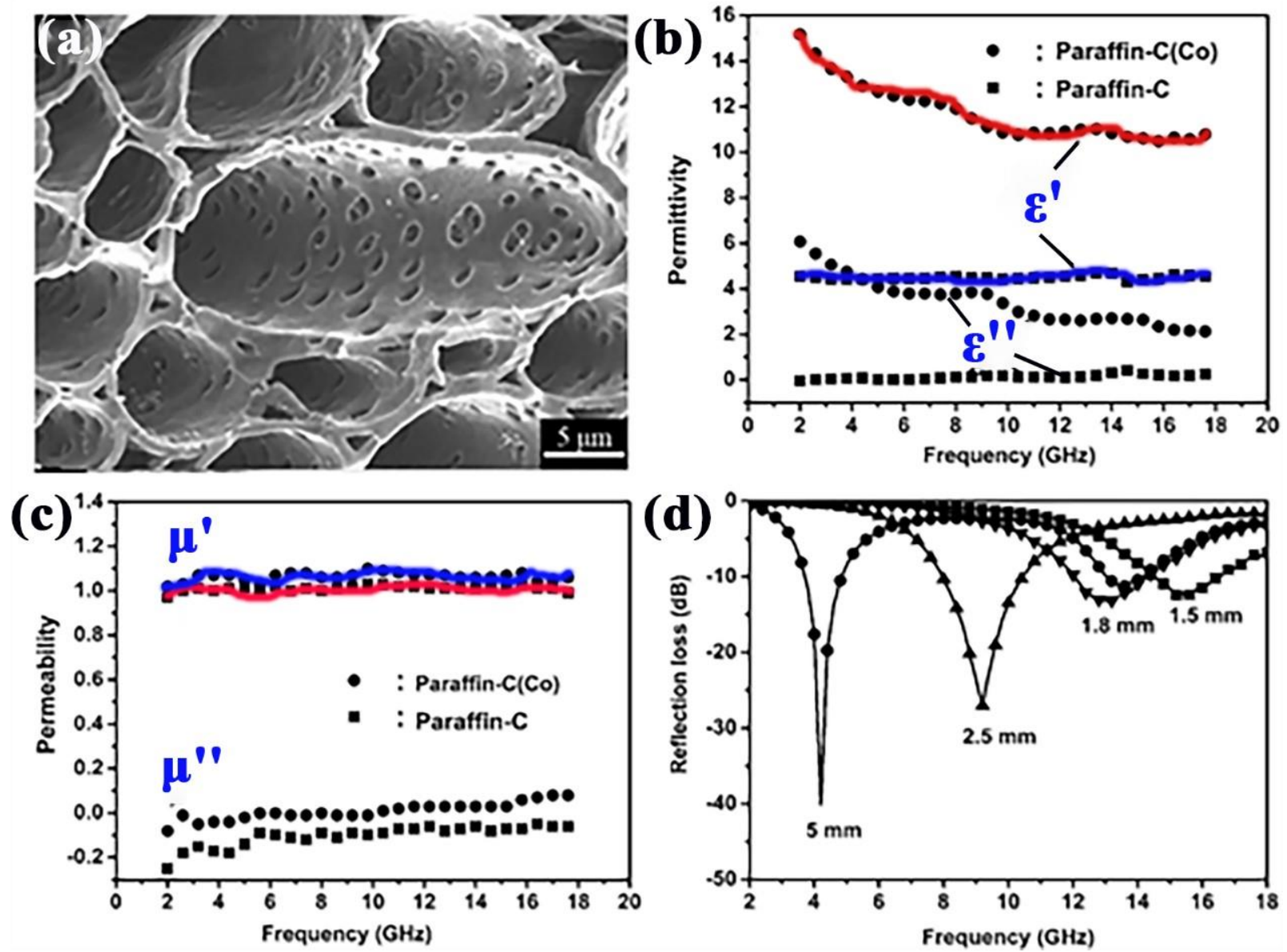

Fig. 6 (a) SEM image of porous carbon/Co nanocomposites; Frequency dependence of (b) complex permittivity and (c) complex permeability (d) reflection loss curves for the porous carbon/Co nanocomposites, reproduced with the Permission from [14].

$\mathrm{dB}$ corresponding to $1.5 \mathrm{~mm}$. The outstanding EMW absorbing ability is mainly resulted from a better impedance matching of Co hollow structures (Fig. 4d). Owing to the existence of abundant air, the permittivity level of the hollow structures can be modulated effectively, which is favorable to obtain an improved impedance matching. The porous threedimensional flower-like $\mathrm{Co} / \mathrm{CoO}$ structures assembled by abundant flakes were synthesized by Lv et al. and the EMW absorption properties were evaluated (Fig. 5a-d). ${ }^{[20]}$ The results indicated that an optimal RL of $-50 \mathrm{~dB}$ and an effective bandwidth covering from 13.8 to $18 \mathrm{GHz}$ was obtained. The excellent EMW absorption of the porous $\mathrm{Co} / \mathrm{CoO}$ flowers was mainly aroused from the high impedance matching. Meanwhile, the numerous pores and defects on the flakes served as polarization centers, providing strong dielectric loss. In addition, the 3D structure was beneficial to generate multiscatterings, further improving the EMW absorbing ability. 

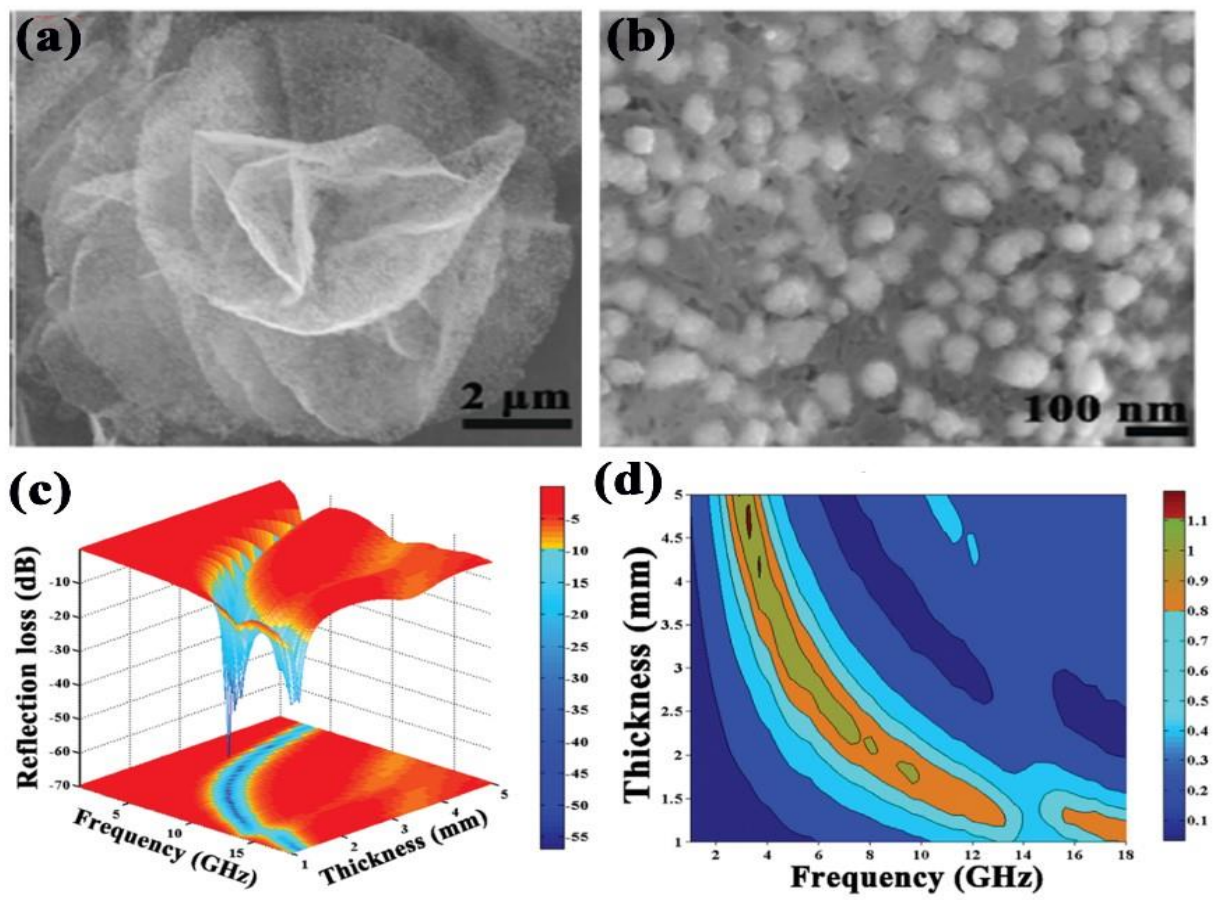

Fig. 7 (a, b) SEM images of the $\mathrm{Co} / \mathrm{C}$ crabapples with different magnetizations; (c) frequency and thickness dependent RL values; (d) impedance matching value in the frequency range of 1-18 GHz, reproduced with the Permission from [27].
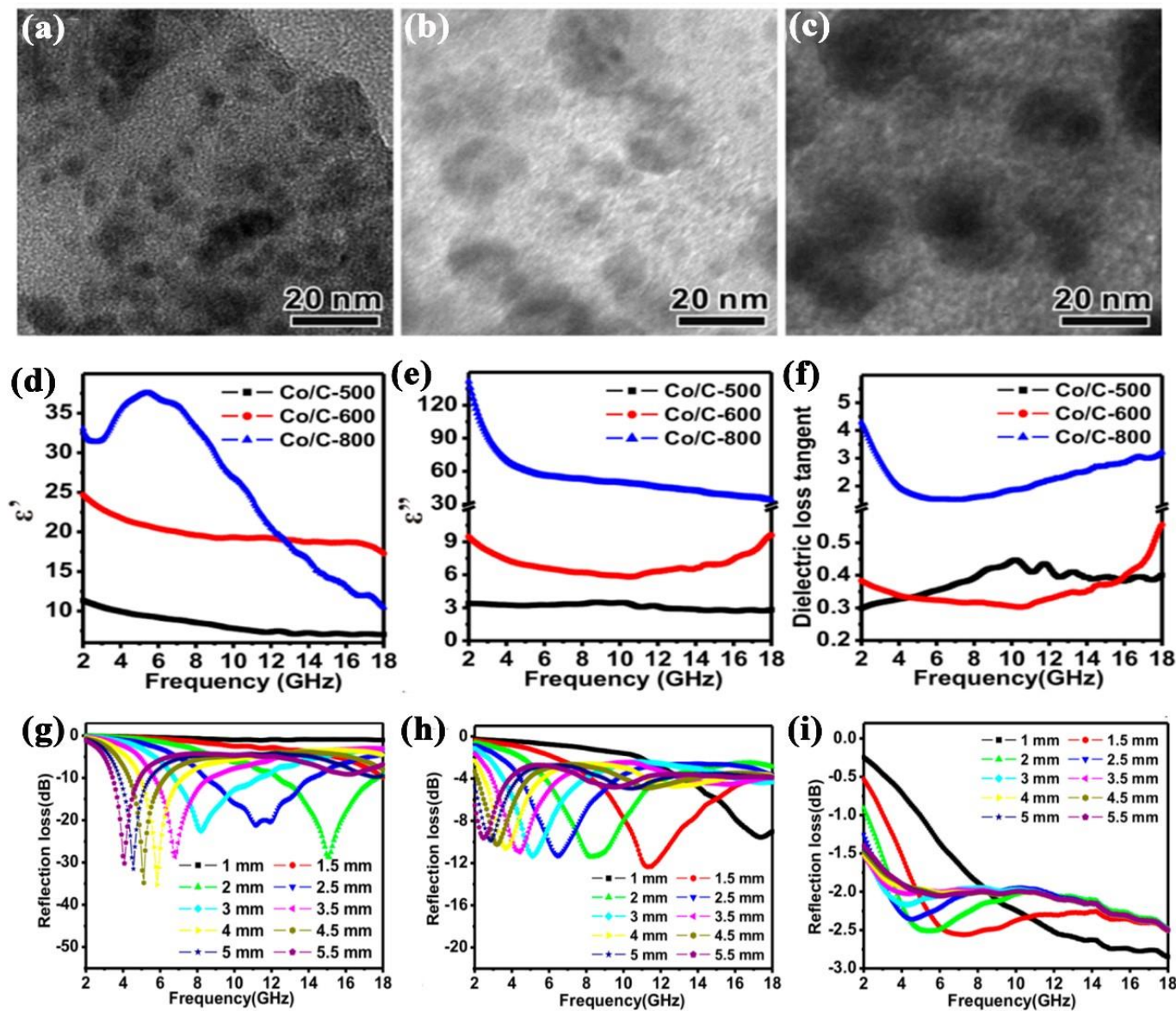

Fig. 8 Low-magnification TEM image of MOFs derived $\mathrm{Co} / \mathrm{C}$ composites obtained at different calcination temperature: (a) $\mathrm{Co} / \mathrm{C}$ 500, (b) $\mathrm{Co} / \mathrm{C}-600$, (c) $\mathrm{Co} / \mathrm{C}-800$; Frequency dependence of (d) permittivity, (e) permeability and (f) dielectric loss tangent for MOFs derived $\mathrm{Co} / \mathrm{C}$ composites obtained at different calcination temperature; Reflection loss values with frequency and thickness for the MOFs derived $\mathrm{Co} / \mathrm{C}$ composites obtained at different calcination temperature: (g) $\mathrm{Co} / \mathrm{C}-500$, (h) $\mathrm{Co} / \mathrm{C}-600$, (i) $\mathrm{Co} / \mathrm{C}-700$, reproduced with the Permission from [29]. 

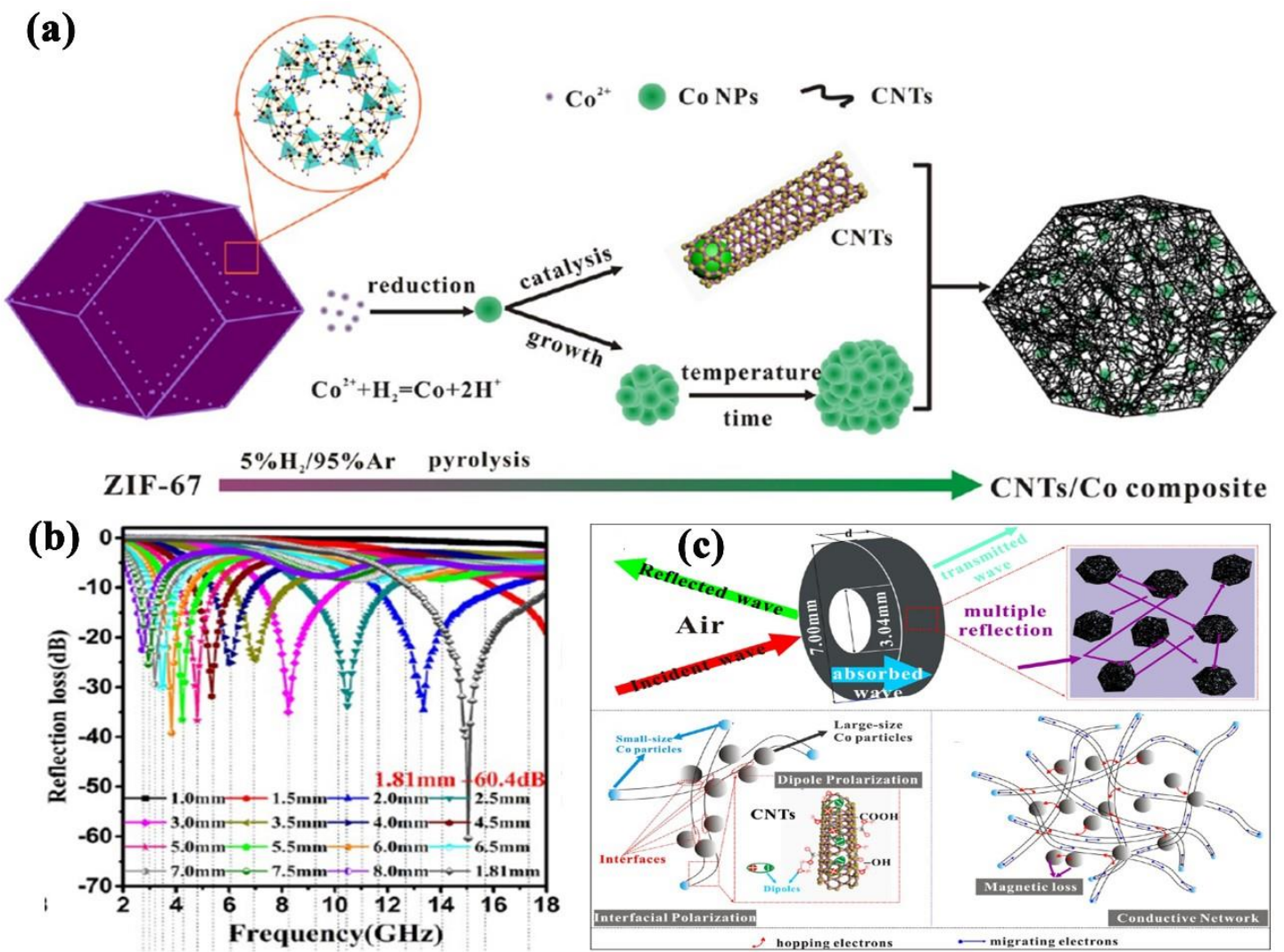

Fig. 9 (a) Schematic presentation of the formation process for CNTs/Co composite; (b) Reflection loss values with frequency and thickness for CNTs/Co composite; (c) Scheme of the attenuation mechanisms for CNTs/Co absorber, reproduced with the Permission from [55].

Above all, for pure $\mathrm{Co}$, the great magnetic properties enable it possess excellent magnetic loss ability. However, the permittivity of pure Co materials is much higher than their permeability, which means their impedance matching level is out of balance. Thus the absorption bandwidth of pure Co is quite narrow. Designing porous structures or hollow structures is an effective way to adjust the permittivity and reach balanced impedance matching. In this way the EMW absorption properties of pure Co materials could be optimized.

\subsection{Co/carbon materials nanocomposites as absorbers}

Although pure Co materials can meet the requirements of strong absorption, the drawbacks of large density and easy to oxidize restrict their applications. Recently, carbon materials including carbon nanotubes (CNTs), carbon nanofibers (CNF), graphene and porous carbon et al, have been reported as the most attractive EMW absorbers owing to their lightweight, high surface area, excellent physical properties and natural abundance. ${ }^{[51-54]}$ When constructing composites of carbon materials and Co, ideal EMW absorbers with characteristics of low density, strong absorption, and broad bandwidth can be obtained owing to the effective combination of dielectric loss and magnetic loss. In this section, we will focus on the progress of $\mathrm{Co} /$ carbon nanocomposites as absorbers, such as $\mathrm{Co} / \mathrm{CNTs}, \mathrm{Co} /$ graphene, Co/porous carbon and $\mathrm{Co} / \mathrm{CNF}$ etc. ${ }^{[34,55-57]}$ The influences of graphitization degree on the EM parameters are discussed in detail.

\subsubsection{Co/porous carbon nanocomposites}

Porous carbon materials are considered as promising EMW absorption absorbers related to their unique structures. Firstly, the porous carbon materials own smaller dielectric constants, meanwhile, they have several times larger dielectric loss compared with their corresponding solid carbons; thus, they exhibit better impedance matching. ${ }^{58}$ Secondly, the large specific surface area of the carbon materials facilitates the high loading of magnetic particles, which is favorable to improve magnetic loss. Thirdly, the porous carbons with low densities can be explored as lightweight EMW absorbers.

Liu reported the preparation of porous carbon/Co nanocomposites through a sol-gel method. ${ }^{[14]}$ Fig. 6 a shows numerous pores with diameters of 1-2 $\mu \mathrm{m}$ were distributed on the porous carbon and Co nanoparticles were encapsulated into carbon shell. Through combination of Co nanoparticles, the porous carbon/Co nanocomposite exhibited larger dielectric loss resulting from the enhanced interfacial polarization and dipole polarization of the core-shell structures (Fig. 6b). The permeability was also increased due to the introduction of magnetic Co particles, which implied the improved magnetic loss of porous carbon/Co nanocomposites. With an effective combination of carbon and Co particles as well as the porous structural advantages, the porous carbon/Co 
composite absorbers reached $\mathrm{RL}_{\min }$ of $-40 \mathrm{~dB}$ at $4.2 \mathrm{GHz}$ with matching thickness of $5 \mathrm{~mm}$. Wu synthesized the hierarchical porous $\mathrm{Co} / \mathrm{C}$ composite crabapples through a facile solvothermal method and a following carbon reduction process. ${ }^{[27]}$ The numerous Co nanoparticles with carbon coating were observed to distribute on the crabapple-like porous carbon frameworks (Fig. 7a and b). Thanks to these unique structural features and synergy of multiple components, the hierarchical porous $\mathrm{Co} / \mathrm{C}$ crabapples displayed significantly enhanced EMW absorbing ability. The $\mathrm{RL}_{\min }$ was as strong as $-56.9 \mathrm{~dB}$ and an effective bandwidth of $5.9 \mathrm{GHz}$ at $1.4 \mathrm{~mm}$ was achieved (Fig. 7c). The absorbing mechanisms were investigated systematically. Natural resonance and exchange resonance were the main cause for magnetic loss and interfacial polarization contributed to the dielectric loss. The impedance matching level was also greatly improved due to the combination of carbon and Co, as shown in Fig. $7 \mathrm{~d}$. The impedance matching value of the composites is associated with the frequency and thickness. It can be observed from Fig. $7 \mathrm{~d}$ that the impedance matching value is between $0.8-1.1$ in a wide frequency range, thus leading to broad-band absorption. Recently, metal-organic-frameworks (MOFs) derived porous carbon with ultrahigh surface area, easy operation, low cost and lightweight has been emerged as attractive candidate for EMW absorption. ${ }^{[59-61]} \mathrm{Lv}$ fabricated the porous $\mathrm{Co} / \mathrm{C}$ nanocomposites successfully through calculation of Co-based MOFs (ZIF-67) under different temperatures (Co/C-500, $\mathrm{Co} / \mathrm{C}-600, \mathrm{Co} / \mathrm{C}-800$, respectively). ${ }^{[29]}$ In this work, the $\mathrm{Co}$ nanoparticles with different sizes were observed to encapsulate into the carbon matrix uniformly $(6 \mathrm{~nm}$ for $\mathrm{Co} / \mathrm{C}$ $500,10 \mathrm{~nm}$ for $\mathrm{Co} / \mathrm{C}-600,20 \mathrm{~nm}$ for $\mathrm{Co} / \mathrm{C}-800$, Fig. 8a-c). It was figured out that the calculation temperature has a great impact on the permittivity level through affecting the graphitization degree of carbon matrix and crystallites of $\mathrm{Co}$ nanoparticles. As shown in Fig. 8d-f, the permittivity of the $\mathrm{Co} / \mathrm{C}$ nanocomposites enhanced with an increase of the calculation temperature, thus tunable EMW absorption performances could be obtained (Fig. 8g-i). Among the samples, the $\mathrm{Co} / \mathrm{C}-500$ nanocomposites exhibited the most excellent EMW absorption properties with the $\mathrm{RL}_{\min }$ of -35.3 $\mathrm{dB}$ at $2.5 \mathrm{~mm}$ and the $f_{e}$ covered from 8.4-14.2 GHz. The results indicated that the MOFs derived porous carbon composites can be the promising candidates for $\mathrm{EM}$ absorption. Li et al reported porous hollow $\mathrm{Co} / \mathrm{C}$ microspheres through MOFs-derived strategy. ${ }^{[30]}$ Due to the improved dielectric loss and matched impedance related to the hollow porous structures, the hollow $\mathrm{Co} / \mathrm{C}$ microspheres showed enhanced EMW absorption comparing with other $\mathrm{Co} / \mathrm{C}$ composites. Wang et al prepared the synthesis of $\mathrm{Co}-\mathrm{C}$ coreshell composite with cobalt cores encapsulated into porous carbon shell deriving from pyrolysis of Co-MOF-74. ${ }^{[62]}$ As an absorber, the $\mathrm{RL}_{\text {min }}$ could attain $-62.12 \mathrm{~dB}$ at $11.85 \mathrm{GHz}$ and RL values less than $-10 \mathrm{~dB}$ was observed in the frequency range of 4.1-18 GHz. The highly efficient EMW absorption was attribute to the synergy of magnetic loss from Co core and high dielectric loss of carbon shell along with the interface polarization due to the core-shell structure. In Wang's report, the Co NPs/porous carbon composites derived from MOFs possessed strong RL of $-30.31 \mathrm{~dB}$ at $11.03 \mathrm{GHz}$ and broad $f_{e}$ of $4.93 \mathrm{GHz}$ was observed. ${ }^{28}$ The EM parameters and absorbing performances can be adjusted effectively through controlling the carbonization temperature. Furthermore, due to the existence of lightweight carbon, the filling ratio of the $\mathrm{Co}$ $\mathrm{NPs} /$ porous carbon composites into paraffin was lowered to 25 $\mathrm{wt} \%$.

\subsubsection{Co/graphene nanocomposites}

As a new kind of carbon material, graphene (GN), composing of sp2-bonded carbon atoms, has become competitive candidate in searching of lightweight and high-efficiency absorbers because of its extraordinary electrical, thermal and mechanical properties. ${ }^{[63,64]}$ More importantly, the threedimensional layered structure of graphene with larger surface area could enable the occurrence of various kinds of polarizations (dipole polarization, interface polarizations, etc), leading to an enhanced dielectric loss ability. ${ }^{[65]}$ In this part, we focused on the development of EMW absorption properties of Co/graphene composites.

Two kinds of $\mathrm{Co} / \mathrm{GN}$ composites with hexagonal closepacked cobalt $(\alpha-\mathrm{Co})$ nanocrystals and face-centered cubic cobalt $(\beta-\mathrm{Co})$ nanospheres deposited on GN nanosheets have been synthesized via one-step solution-phase method. ${ }^{[34]}$ The as-synthesized $\alpha-\mathrm{Co} / \mathrm{GN}$ and $\beta-\mathrm{Co} / \mathrm{GN}$ composites presented enhanced EMW absorbability than pure Co nanocrystals or graphene. For the composites of $\alpha-\mathrm{Co} / \mathrm{GN}$ absorber, the strongest RL peak reached $-47.5 \mathrm{~dB}$ at $11.9 \mathrm{GHz}$ with a thin thickness of $2 \mathrm{~mm}$ and this value was superior to previously reported $\mathrm{Fe}_{3} \mathrm{O}_{4} / \mathrm{GN}$ composites. Zeng reported the synthesis of hollow reduced graphene oxide microspheres embedded with Co nanoparticles (Air@rGO@Co). ${ }^{[66]}$ Attributing to the coeffect of magnetic loss from $\mathrm{Co}$ and dielectric effect from $\mathrm{rGO}$, the Air@rGO@Co composites showed quite low $\mathrm{RL}_{\min }$ of $68.1 \mathrm{~dB}$ at $13.8 \mathrm{GHz}$ with the thickness of $2.2 \mathrm{~mm}$ and the corresponding bandwidth achieved $7.1 \mathrm{GHz}$. What's more, the EMW absorption performance can be controlled effectively through adjusting the ratio of $\mathrm{Co}$ and rGo. Long reported cobalt nanoparticle-graphene nanocomposite was fabricated through a facile method and the as-synthesized CoNP-G nanocomposite provided effective EMW absorption in the frequency range of $2.4-3.84,7.84-11.87$ and $13.25-18 \mathrm{GHz}$, respectively. ${ }^{[67]}$ The magnetic loss from cobalt and dielectric loss from graphene contributed synergistically to a strong RL of $-40.53 \mathrm{~dB}$.

\subsubsection{Co/carbon nanotubes (CNTs) and nanofibers (CNF) nanocomposites}

Wu et al has synthesized the Co nanoparticles $(20-30 \mathrm{~nm})$ decorated MWCNTs (Co/MWCNTs) nanocomposites through combining the hydrothermal method with following carbon reduction process. ${ }^{[56]}$ Comparisons of the EMW absorption 
Table 1. EMW absorption properties of previously reported pure Co materials and $\mathrm{Co} / \mathrm{C}$ composites.

\begin{tabular}{|c|c|c|c|c|c|c|}
\hline \multirow{2}{*}{ Absorber } & \multirow{2}{*}{$\begin{array}{l}\text { Filler } \\
\text { raio }\end{array}$} & \multirow{2}{*}{$\begin{array}{l}\text { Optimal RL } \\
\qquad(\mathrm{dB})\end{array}$} & \multirow{2}{*}{$\begin{array}{l}\text { Optimal thickness } \\
\qquad(\mathrm{mm})\end{array}$} & \multicolumn{2}{|c|}{ Bandwidth } & \multirow[b]{2}{*}{ Ref. } \\
\hline & & & & Thickness(mm) & $\mathrm{RL}<-10 \mathrm{~dB}(\mathrm{GHz})$ & \\
\hline Co nanochains & $20 \mathrm{wt} \%$ & -17.0 & 3.0 & 3.0 & 2.0 & [39] \\
\hline Co nanowires & $50 \mathrm{wt} \%$ & -23.5 & 5.0 & 2.0 & --- & [40] \\
\hline Co nanoplates & 13 vol\% & -22.5 & 4.0 & 2.0 & 1.0 & [43] \\
\hline Co particles & $70 \mathrm{wt} \%$ & -19.0 & 5.0 & 5.0 & 2.0 & [70] \\
\hline Co dendrites & $65 \mathrm{wt} \%$ & -35.6 & 3.0 & 2.0 & 3.0 & [19] \\
\hline $\begin{array}{c}\text { Nanosheet hierarchical Co } \\
\text { particles }\end{array}$ & $70 \mathrm{wt} \%$ & -54.9 & 1.5 & 2.0 & 3.8 & [71] \\
\hline Sword-like Co particles & $70 \mathrm{wt} \%$ & -60.13 & 1.4 & 2.0 & 2.7 & [44] \\
\hline Flower-like Co structures & $67 \mathrm{wt} \%$ & -40.25 & 2.5 & 2.0 & 2.3 & [22] \\
\hline Porous $\mathrm{Co} / \mathrm{CoO}$ flower & $50 \mathrm{wt} \%$ & -50 & 3.5 & 2.0 & 4.2 & [20] \\
\hline Porous $\mathrm{Co} / \mathrm{C}-500$ & $40 \mathrm{wt} \%$ & -35.3 & 4.0 & 2.0 & 5.5 & [29] \\
\hline Porous carbon/Co & $30 \mathrm{wt} \%$ & -40.0 & 5.0 & 1.8 & 2.5 & [14] \\
\hline Porous $\mathrm{Co} / \mathrm{C}-800$ & $30 \mathrm{wt} \%$ & -39.6 & 2.55 & 2.0 & 3.8 & [72] \\
\hline Porous $\mathrm{Co} / \mathrm{C}$ crabapples & $30 \mathrm{wt} \%$ & -26.6 & 1.84 & 2.0 & 5.8 & [27] \\
\hline $\mathrm{Co} / \mathrm{C}$ nanoparticles & $50 \mathrm{wt} \%$ & -43.4 & 2.3 & 2.0 & 8.0 & [69] \\
\hline Co@C microspheres & $70 \mathrm{wt} \%$ & -68.7 & 1.65 & 2.0 & 2.7 & [73] \\
\hline$\alpha$-Co/GN nanocomposites & $60 \mathrm{wt} \%$ & -47.5 & 2.0 & 2.0 & 6.0 & [34] \\
\hline Co NP-G nanocomposite & $55 \mathrm{wt} \%$ & -40.53 & 4.5 & 2.0 & 3.5 & [67] \\
\hline Co@N-C composites & $30 \mathrm{wt} \%$ & -18.03 & 1.8 & 2.1 & 4.2 & [74] \\
\hline Co/CNTs nanocomposites & $20 \mathrm{wt} \%$ & -36.5 & 4.0 & 2.1 & 2.0 & [56] \\
\hline Porous CNTs/Co composite & $20 \mathrm{wt} \%$ & -60.4 & 1.81 & 2.0 & 5.0 & [55] \\
\hline $\begin{array}{l}\text { Co-C/MWCNTs } \\
\text { nanocomposites }\end{array}$ & $25 \mathrm{wt} \%$ & -50.0 & 2.4 & 1.8 & 4.3 & [61] \\
\hline CNF-Co composite nanofibers & $5 \mathrm{wt} \%$ & -63.1 & 1.6 & 2.0 & 6.2 & [57] \\
\hline
\end{tabular}

properties for pure MWCNTs and Co/MWCNTs absorption than pure CNTs or Co nanoparticles due to an nanocomposites were carried out. Due to the effective improved impedance matching of CNTs/Co composites. ${ }^{[68]}$ combination of strong magnetic loss from $\mathrm{Co}$ and dielectric More recently, Yin and co-workers proposed the preparation loss from MWCNTs as well as a better impedance matching, of CNTs/Co composite with dodecahedron morphology the Co/MWCNTs nanocomposites exhibited a greatly through pyrolysis of the Co-based zeolitic imidazolate enhanced EMW absorption than the pure CNTs and the framework under $\mathrm{Ar} / \mathrm{H}_{2}$ atmosphere (Fig. 9a). ${ }^{[55]}$ The pyrolysis strongest absorption peak reached $-36.5 \mathrm{~dB}$. Sui demonstrated temperature was found to have a great influence on the that the CNTs/Co composites possessed higher EMW graphitization of CNTs and crystallinity of Co nanoparticles. 
With a low filler loading of only $20 \mathrm{wt} \%$ in the paraffin wax, the $\mathrm{CNTs} / \mathrm{Co}$ composite achieved $\mathrm{RL}_{\text {min }}$ of $-60.4 \mathrm{~dB}$ locating at ultrathin thickness of $1.81 \mathrm{~mm}$, and the results were displayed in Fig. 9b. The strong broadband absorbing performance is mainly originated from the strong dielectric loss caused by the interfacial polarization, dipole polarization, and hopping of electrons. The magnetic loss is mainly from natural resonance and exchange resonance of Co NPs. Besides, the multiple scatterings and reflections among $\mathrm{CNTs} / \mathrm{Co}$ particles further promoted the attenuation ability through increasing the traveling routes of incident EM waves. Xiang prepared the magnetic carbon nanofibers containing uniformly dispersed Co nanoparticles (ca. $155 \mathrm{~nm}$ ) as highly-efficient absorbers, which showed optimal RL of $-63.1 \mathrm{~dB}$ with matching thickness of $1.6 \mathrm{~mm} .{ }^{[57]}$ Moreover, the effective absorption frequency range covers from 4 to $18 \mathrm{GHz}$ over thickness ranges of $1.1-5.0 \mathrm{~mm}$ ascribed to a complementary effect of CNF and Co particles.

Above all, various $\mathrm{Co} / \mathrm{C}$ composites were fabricated, and the carbon components include CNTs, CNF, graphene and porous carbon. The $\mathrm{Co} / \mathrm{C}$ composites showed enhanced impedance matching and absorption properties than single components. Particularly, Co/porous carbon composites could reach the requirements of both lightweight and broad absorption bandwidth, which would be the best candidate for ideal absorbents.

\section{Comparisons of EMW absorption performance for pure $\mathrm{Co}$ and $\mathrm{Co} / \mathrm{C}$ composites}

As discussed above, there are several factors influencing the EMW absorption performances of the pure $\mathrm{Co}$ and $\mathrm{Co} / \mathrm{C}$ composite absorbers, such as the absorber components, filler content, microstructures and graphitization degree. In order to figure out a general index for construction of ideal Co based absorbers, as shown in Table 1, we carried out comparisons of EMW absorption properties for pure $\mathrm{Co}$ and $\mathrm{Co} / \mathrm{C}$ composites in terms of filling ratio, optimal RL value, optimal thickness and effective absorbing bandwidth at $2.0 \mathrm{~mm}$ thickness.

Comparing with $1 \mathrm{D}$ or $2 \mathrm{D}$ Co materials, the $\mathrm{Co} / \mathrm{C}$ composite absorbers exhibited great superiorities. Although the 3D dendrite-like or flower-like Co can display strong absorbing capability, the filling ratio is always over $60 \mathrm{wt} \%$ due to the large density of magnetic metals. Actually, the filling ratio of absorbers plays an important role in determining the EMW absorption performances. This is because the matching between dielectric loss and magnetic loss can be greatly influenced through adjusting the filling ratios. Liu investigated the effects of filling ratios on the EMW absorption properties for $\mathrm{Co} / \mathrm{C}$ composites. ${ }^{[69]}$ In Liu's work, the $\mathrm{Co} / \mathrm{C}$ NPs were mixed in paraffin wax with filling ratio of 25,50 and $75 \mathrm{wt} \%$, receptively, and their absorption performance were discussed in 2-18 GHz. When the filling ratio is $50 \mathrm{wt} \%$, the best EMW absorption can be achieved with the strongest RL of $-43.4 \mathrm{~dB}$. The EMW absorption performance would be decreased if further increasing the filling ratio. Through calculating the magnetic/dielectric loss tangent, it was observed that the composites containing $50 \mathrm{wt}$ $\% \mathrm{Co} / \mathrm{C}$ NPs exhibited the best impedance matching among the samples. The attenuation mechanisms changed from magnetic loss to dielectric loss with a decrease of filling ratio in the matrix. High filling ratio will result in a rather high permittivity, leading to weak absorption. As expected, the introduction of lightweight porous carbon, carbon nanotubes and carbon nanofibers could effectively decrease the filling ratio of the composites together with highly efficient EMW absorption, as presented in Table 1.

Comparing with 1D Co nanowires, Co nanochains, Co particles or 2D Co nanoplates, the Co absorbers composed of 3D flake-like structure or porous structure exhibited more superiorities (Table 1). The superiorities of 3D Co-based absorbers compared with 1D and 2D structures were reflected by the enhanced polarization loss and multi-reflection effect.

\section{Conclusions and Perspectives}

In this review, we summarized the development of Co based EMW absorbers, including pure Co nanostructures with different morphologies and various $\mathrm{Co} / \mathrm{C}$ nanocomposites. The microstructures, filling ratios, and graphitization degree were found to have a great influence on the EMW absorption performances. In specific, Co absorbers with 1D chain-like structure, 2D flake-like structure and 3D flower-like structure were fabricated and their EMW absorption properties were investigated. The conclusions can be drawn as follows:

1. Compared with $1 \mathrm{D}$ or $2 \mathrm{D}$ materials, the flakeassembled structure is much favorable because the large shape anisotropy of flake-like structure can exceed Snoek's limit and thus a high permeability can be obtained in $\mathrm{GHz}$ range. Besides, the 3D or porous structure with large surface area possess massive sites, which can act as polarized centers, enhancing the dielectric loss.

2. Compared with pure $\mathrm{Co}$ materials, the $\mathrm{Co} / \mathrm{C}$ composites exhibited enhanced impedance matching and EMW absorption than single component. However, the permittivity can be greatly affected by the graphite degree and tunable EMW absorption would be obtained with the variation of graphitization degree. Therefore, fabricating Co based absorbers with ideal compositions and optimal structure will be target direction to obtain significantly enhanced EMW absorption properties in the future.

3. Fabricating composites of cobalt and porous carbon through MOFs derived method exhibit advantages of lightweight, tunable EMW absorption and controllable synthetic process, showing significant potential as promising EMW absorbers.

Despite the above achievements, there are far more 
problems needed to be investigated. Firstly, the intrinsic absorbing mechanisms for magnetic Co particles and their composites are still not clear. Most of the current researches are focused on the construction of different components and microstructures to enhance the microwave absorption properties. However, a lot of questions were still existed. For example, how did the microstructure influence the resonance frequency? What is the accurate occurring frequency for different polarizations? How did the interface polarization affect the overall conductivity? Systematic investigations are urgently needed to address these problems. Secondly, the current articles have proved that 3D structures is helpful to enhance the attenuation ability owing to the multi-scattering and reflections effect. But the exact contributions from multiscattering and reflections to EM wave absorption can't be evaluated. Thirdly, attaining thin thickness in low frequency range is quite difficult. According to the quarter-wavelength model $\left(d=n \lambda / 4=n c /\left(4 f \sqrt{\left|\mu_{r}\right|\left|\varepsilon_{r}\right|}\right)\right)$, the thickness is inversely proportional to frequency, module value of permittivity and permeability. It means that in order to get thin thickness in low frequency, permittivity must be improved. At the same time, the permittivity level is restricted by the impedance matching. Thus, to explore an ideal method to reach a balance between improving the permittivity and achieving impedance matching is quite essential. In conclusion, there remains much to learn about the magnetic/dielectric loss mechanisms and thickness optimization. We believe that this review could provide a future direction for research of Co based materials as EM wave absorbers.

\section{Acknowledgements}

The authors acknowledge the support from Shandong University of Science and Technology research fund (01040125215).

\section{Support information}

Not applicable

\section{Conflict of Interest}

There are no conflicts to declare.

\section{References}

[1] B. Zhao, J. Deng, R. Zhang, L. Liang, B. Fan, Z. Bai, G. Shao, C. Park, Eng. Sci., 2018, 3, 5-40, doi: 10.30919/es8d735.

[2] H. Hu, H. Liu, D. Zhang, J. Wang, G. Qin, X. Zhang, Eng. Sci., 2018, 2, 43-48, doi: 10.30919/es8d136.

[3] L. Lv, J. Liu, H. Liu, C. Liu, Y. Lu, K. Sun, R. Fan, N. Wang, N. Lu, Z. Guo, E. Wujcik, Eng. Sci., 2018, 2, 26-42, doi:10.30919/es8d615.

[4] L. Kong, X. Yin, X. Yuan, Y. Zhang, X. Liu, L. Cheng, L. Zhang, Carbon, 2014, 73, 185-193, doi: 10.1016/j.carbon.2014.02.054.

[5] J. R. Liu, M. Itoh, K. Machida, Appl. Phys. Lett., 2003, 83, 4017-4019, doi: 10.1063/1.1623934.
[6] D. Xu, N. Wu, K. Le, F. Wang, Z. Wang, L. Wu, W. Liu, A. Ouyang, J. Liu, J. Mat. Chem. C, 2020, 8, 2451-2459, doi: 10.1039/C9TC05852D.

[7] S. Gupta, N.-H. Tai, Carbon, 2019, 152, 159-187, doi: 10.1016/j.carbon.2019.06.002.

[8] H. Wu, M. Qin, L. Zhang, Compos. B. Eng., 2020, 182, 107620, doi: 10.1016/j.compositesb.2019.107620.

[9] J. Liu, H. Liang,Y. Zhang, G. Wu, H. Wu, Compos.B.Eng., 2019, 176, 107240, doi: 10.1016/j.compositesb.2019.107240.

[10] X. Zhang, J. Qiao, J. Zhao, D. Xu, F. Wang,C. Liu, Y. Jiang, L. Wu, P. Cui, L. Lv, Q. Wang, W. Liu, Z. Wang, J. Liu, ACS Appl. Mater. Interf., 2019, 11, 35959-35968, doi: 10.1021/acsami.9b10168.

[11] J. Qiao, X. Zhang, D. Xu, L. Kong, L. Lv, F. Yang, F. Wang, W. Liu, J. Liu, Chem. Eng. J., 2020, 380, 122591, doi: 10.1016/j.cej.2019.122591.

[12] M.-S. Cao,Y.-Z. Cai, P. He, J.-C. Shu, W.-Q. Cao, J. Yuan, Chem. Eng. J., 2019, 359, 1265-1302,doi: 10.1016/j.cej.2018.11.051.

[13] X. Zhang, G. Ji, W. Liu, B. Quan, X. Liang, C. Shang, Y. Cheng, Y. Du, Nanoscale, 2015, 7, 12932-12942, doi: 10.1039/C5NR03176A.

[14] Q. Liu, D. Zhang, T. Fan, Appl. Phys. Lett., 2008, 93, 013110 , doi: 10.1063/1.2957035.

[15] F. Ma, Y. Qin,Y.-Z. Li, Appl. Phys. Lett., 2010, 96, 202507, doi: 10.1063/1.3432441.

[16] C. He, S. Qiu, X. Wang, J. Liu, L. Luan, W. Liu,M. Itoh, K. Machida, J. Mater. Chem., 2012, 22, 22160-22166,doi: 10.1039/C2JM33068G.

[17] W. Liu, S. Tan, Z. Yang, G. Ji, ACS Appl. Mater. Interfaces, 2018, 10, 31610-31622, doi: 10.1021/acsami.8b10685.

[18] T. Huang, Z. Wu, J. Lin, Q. Yu, D. Tan, L. Li, ACS Appl. Electron. Mater., 2019, 1, 2541-2550, doi: 10.1021/acsaelm.9b00565.

[19] X. Wang, G. Shi, F.-N. Shi, G. Xu, Y. Qi, D. Li, Z. Zhang, Y. Zhang, H. You, RSC Advances, 2016, 6, 40844-40853, doi: 10.1039/C6RA06112E.

[20] H. Lv, X. Liang, G. Ji, H. Zhang, Y. Du, ACS Appl. Mater. Interfaces, 2015, 7, 9776-9783, doi: 10.1021/acsami.5b01654.

[21] L. J. Deng, P. H. Zhou, J. L. Xie, L. Zhang, J. Appl. Phys, 2007, 101, 103916, doi: 10.1063/1.2733610.

[22] G. Tong, J. Yuan, W. Wu, Q. Hu, H. Qian, L. Li, J. Shen, Cryst. Eng. Comm., 2012, 14, 2071, doi: 10.1039/C2CE05910J.

[23] Q. Liu, Q. Cao, H. Bi, C. Liang, K. Yuan, W. She, Y. Yang, R. Che, Adv. Mater, 2016, 28, 486-490, doi: 10.1002/adma.201503149.

[24] H. Wang, Z. Yan, J. An, J. He, Y. Hou, H. Yu, N. Ma, G. Yu, D. Sun, $R S C \quad A d v ., \quad 2016, \quad 6, \quad 92152-92158$, doi: 10.1039/C6RA16003D.

[25] D. Zhang, F. Xu, J. Lin, Z. Yang, M. Zhang, Carbon, 2014, 80, 103-111, doi: 10.1016/j.carbon.2014.08.044.

[26] T. Liu, Y. Pang, M. Zhu, S. Kobayashi, Nanoscale, 2014, 6, 2447, doi: 10.1039/C3NR05238A.

[27] N. Wu, C. Liu, D. Xu, J. Liu, W. Liu, H. Liu, J. Zhang, W. Xie, Z. Guo, J. Mater. Chem. C, 2019, 7, 1659-1669, doi: 
10.1039/C8TC04984J.

[28] H. Wang, L. Xiang, W. Wei, J. An, J. He, C. Gong, Y. Hou, ACS Appl. Mater. Interfaces, 2017, 9, 42102-42110, doi: 10.1021/acsami.7b13796.

[29] Y. Lü, Y. Wang, H. Li, Y. Lin, Z. Jiang, Z. Xie, Q. Kuang, L. Zheng, ACS Appl. Mater. Interfaces, 2015, 7, 13604-13611, doi: 10.1021/acsami.5b03177.

[30] Z. Li, X. Han, Y. Ma, D. Liu, Y. Wang, P. Xu, C. Li, Y. Du, ACS Sustain. Chem. Eng., 2018, 6, 8904-8913, doi:10.1021/acsami.5b03177.

[31] X. F. Zhang, X. L. Dong, H. Huang, Y. Y. Liu, W. N. Wang, X. G. Zhu, B. Lv, J. P. Lei, Lee, C. G., Appl. Phys. Lett., 2006, 89, 053115. doi: 10.1063/1.2236965.

[32] J. R. Liu, M. Itoh, K. Machida, Appl. Phys. Lett., 2006, 88, 062503, doi: 10.1063/1.2170402.

[33] Q. Liao, M. He, Y. Zhou, S. Nie, Y. Wang, S. Hu, H. Yang, H. Li, Y. Tong, ACS Appl. Mater. Interfaces, 2018, 10, 29136 29144, doi: 10.1021/acsami.8b09093.

[34] G. Pan, J. Zhu, S. Ma, G. Sun, X. Yang, ACS Appl. Mater. Interfaces, 2013, 5, 12716-12724, doi: 10.1021/am404117v.

[35] N. Wu, C. Liu, D. Xu, J. Liu, W. Liu, Q. Shao, Z. Guo, ACS Sustain. Chem. Eng., 2018, 6, 12471-12480, doi: 10.1021/acssuschemeng.8b03097.

[36] H. Lv, X. Liang, Y. Cheng, G. Ji, D. Tang, B. Zhang, H. Zhang, Y. Du, RSC Advances, 2015, 5, 25936-25941, doi: 10.1039/C5RA00598A.

[37] S. Qiu, H. Lyu, J. Liu, Y. Liu, N. Wu, W. Liu, ACS Appl. Mater. Interfaces, 2016, 8, 20258-20266, doi: 10.1021/acsami.6b03159.

[38] Z. An, J. Zhang, J.Mat.Chem.C, 2016, 4, 7979-7988, doi: 10.1039/C6TC02669A.

[39] X.-L. Shi, M.-S. Cao, J. Yuan, X.-Y. Fang, Appl. Phys. Lett., 2009, 95, 163108,doi: 10.1063/1.3250170.

[40] W. Chen, M. Han, L. Deng, Physica B Condens. Matter, 2010, 405, 1484-1488, doi: 10.1016/j.physb.2009.12.026.

[41] R. K. Walser, W. Win, P. M Valanju, IEEE T. Magn.,1998, 34, 1390-1392, doi: 10.1109/20.706558.

[42] R. M. Walser, W. Kang, IEEE T. Magn., 1998, 34, 1144-1146, doi: $10.1109 / 20.706419$.

[43] J. Li, J. Huang, Y. Qin, F. Ma, Mater. Sci. Eng. B, 2007, 138, 199-204, doi: 10.1016/j.mseb.2006.12.001.

[44] S. Wen, X. Zhao, Y. Liu, J. Cheng, H. Li, RSC Adv., 2014, 4, 40456, doi: 10.1039/C4RA05716C.

[45] H. Xu, X. Yin, M. Zhu, M. Han, Z. Hou, X. Li, L. Zhang, L. Cheng, ACS Appl. Mater. Interfaces, 2017, 9, 6332-6341,doi: 10.1021/acsami.6b15826.

[46] P. Liu, V. M. H. Ng, Z. Yao, J. Zhou, Y. Lei, Z. Yang, H. Lv, L. B. Kong, ACS Appl. Mater. Interfaces, 2017, 9, 16404 16416,doi: 10.1021/acsami.7b02597.

[47] X. Sun, J. He, G. Li, J. Tang, T. Wang, Y. Guo, H. Xue, J. Mater. Chem. C, 2013, 1, 765-777, doi: 10.1039/C2TC00159D.

[48] H. Zhao, J. Z. Yeow Seow, Y. Cheng, Z. J. Xu, G. Ji, Ceram. Int., 2020, 46, 15447-15455, doi: 10.1016/j.ceramint.2020.03.089.

[49] T. Liu, P. H. Zhou, J. L. Xie, L. J. Deng, J. Appl. Phys.,
2011, 110, 033918, doi: 10.1063/1.3622144.

[50] C. Wang, X. Han, X. Zhang, S. Hu, T. Zhang, J. Wang, Y. Du, X. Wang, P. Xu, J. Phys. Chem. C, 2010, 114, 14826-14830, doi: 10.1021/jp1050386.

[51] B. Quan, X. Liang, G. Ji, Y. Zhang, G. Xu, Y. Du, ACS Appl. Mater. Interfaces, 2017, 9, 38814-38823, doi: 10.1021/acsami.7b13411.

[52] S. Dai, B. Quan, B. Zhang, X. Liang, G. Ji, Dalton T., 2018, 47, 14767-14773, doi: 10.1039/C8DT03282C.

[53] D. Liu, Y. Du, P. Xu, N. Liu, Y. Wang, H. Zhao, L. Cui, X. Han, J. Mat. Chem. C, 2019, 7, 5037-5046, doi: 10.1039/C9TC00771G.

[54] Y. Wang, X. Gao, Y. Fu, X. Wu, Q. Wang, W. Zhang, C. Luo, Compos. B. Eng., 2019, 169, 221-228, doi: 10.1016/j.compositesb.2019.04.008.

[55] Y. Yin, X. Liu, X. Wei, R. Yu, J. Shui, ACS Appl. Mater. Interfaces, 2016, 8, 34686-34698, doi: 10.1021/acsami.6b12178. [56] N. Wu, H. Lv, J. Liu, Y. Liu, S. Wang, W. Liu, Phys. Chem. Chem. Phys., 2016, 18, 31542-31550, doi: 10.1039/C6CP06066H.

[57] J. Xiang, J. Li, X. Zhang, Q. Ye, J. Xu, X. Shen, J. Mater. Chem. A, 2014, 2, 16905-16914,doi: 10.1039/C4TA03732D.

[58] Z. Fang, C. Li, J. Sun, H. Zhang, J. Zhang, Carbon, 2007, 45, 2873-2879,doi: 10.1016/j.carbon.2007.10.013.

[59] W. Liu, L. Liu, Z. Yang, J. Xu, Y. Hou, G. A. Ji, ACS Appl. Mater. Interfaces, 2018, 10, 8965-8975, doi: 10.1021/acsami.8b00320.

[60] N. Wu, D. Xu, Z. Wang, F. Wang, J. Liu, W. Liu, Q. Shao, H. Liu, Q. Gao, Z. Guo, Carbon, 2019, 145, 433-444, doi: 10.1016/j.carbon.2019.01.028.

[61] R. Shu, W. Li, Y. Wu, J. Zhang, G. Zhang, Chem. Eng. J., 2019, 362, 513-524,doi: 10.1016/j.cej.2019.01.090.

[62] K. Wang, Y. Chen, R. Tian, H. Li, Y. Zhou, H. Duan, H. Liu, ACS Appl. Mater. Interfaces, 2018, 10, 11333-11342, doi: 10.1021/acsami.8b00965.

[63] B. Wen, M. Cao, M. Lu, W. Cao, H. Shi, J. Liu, X. Wang, H. Jin, X. Fang, W. Wang, J. Yuan, Adv. Mater., 2014, 26, 3484 3489,doi:10.1002/adma.201400108.

[64] C. Wang, V. Murugadoss, J. Kong, Z. He, X. Mai, Q. Shao, Y. Chen, L. Guo, C. Liu, S. Angaiah, Z. Guo, Carbon, 2018, 140, 696-733,doi: 10.1016/j.carbon.2018.09.006.

[65] X. Zheng, J. Feng, Y. Zong, H. Miao, X. Hu, J. Bai, X. Li, J. Mat. Chem. C, 2015, 3, 4452-4463, doi: 10.1039/C5TC00313J.

[66] Q. Zeng, P. Chen, Q. Yu, H. Chu, X. Xiong, D. Xu, Q. Wang, Sci. Rep., 2017, 7, 1-9, doi: 10.1038/s41598-017-08293-3.

[67] Q. Long, Z. Xu, H. Xiao, K. Xie, RSC Adv., 2018, 8, 1210 1217, doi: 10.1039/C7RA12190C.

[68] J. Sui, C. Zhang, J. Li, Z. Yu, W. Cai, Mater. Lett., 2012, 75, 158-160,doi: 10.1016/j.matlet.2012.02.007.

[69] T. Liu, X. Xie, Y. Pang, S. Kobayashi, J. Mat. Chem. C, 2016, 4, 1727-1735,doi: 10.1039/C5TC03874J.

[70] S. Wen, Y. Liu, X. C. Zhao, J. W. Cheng, H. Li, Powder 
Technol., 2014, 264, 128-132, doi: 10.1016/j.powtec.2014.05.030.

[71] S. L. Wen, Y. Liu, X. C. Zhao, J. W. Cheng, H. Li, Phys. Chem. Chem. Phys., 2014, 16, 18333,doi: 10.1039/C4CP01468E. [72] R. Qiang, Y. Du, D. Chen, W. Ma, Y. Wang, P. Xu, J. Ma, H. Zhao, X. Han, J. Alloys Compd., 2016, 681, 384-393, doi: 10.1016/j.jallcom.2016.04.225.

[73] D. Ding, Y. Wang, X. Li, R. Qiang, P. Xu, W. Chu, X. Han, Y. Du, Carbon, 2017, 111, 722-732, doi: 10.1016/j.carbon.2016.10.059.

[74] B. Quan, G. Xu, W. Gu, J. Sheng, G. Ji, J. Colloid Interface Sci., 2019, 533, 297-303, doi: 10.1016/j.jcis.2018.08.083.

\section{Author information}

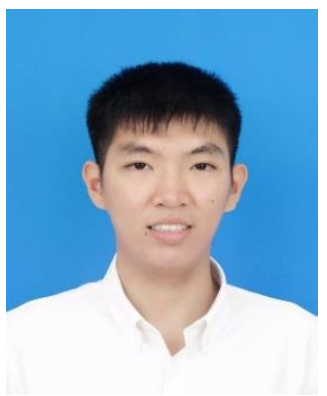

Nannan Wu earned his Ph.D. degree in Materials Science and Engineering from Shandong University in 2020. He is a lecturer in department of Materials Science and Engineering at Shandong University of Science and Technology. His current research interests are in the areas of nanomaterials for energy conversion/storage and electromagnetic interference (EMI) shielding.

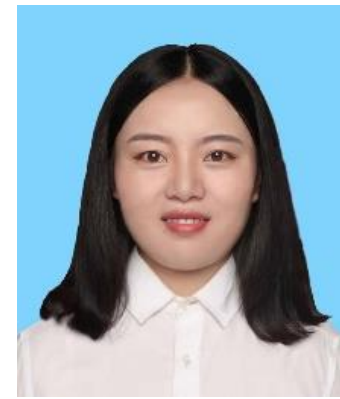

Wenjing Du earned his Ph.D. degree in Materials Science and Engineering from Shandong University in 2020. She is a lecturer in department of College of Materials Engineering at Henan University of Engineering. Her current research interest are in the areas of nano-materials for energy conversion/storage and gas sensors.

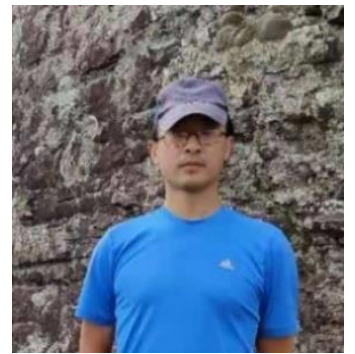

Qinglong Jiang is an Assistant Professor (Tenure Track) in the Department of Chemistry and Physics in University of Arkansas, Pine Bluff. Prior to joining in UAPB, Dr. Jiang worked in Argonne National Lab after his postdoc researcher career in Florida State University. His research focuses on nanomaterials and technologies for electric-optical devices, such as halide perovskite for solar cell and light emitting, dye sensitive solar cell, electrochromism, sensors, fluorescence, etc. He has publications on Adv. Energy Mater., Angew. Chem. Int. Ed., ACS Nano, Nano Energy, ACS Energy Lett., et al.

Publisher's Note: Engineered Science Publisher remains neutral with regard to jurisdictional claims in published maps and institutional affiliations. 